\title{
Design and synthesis of $N$-substituted-2-hydroxyiminoacetamides and interactions with cholinesterases
}

\author{
Nikola Maraković ${ }^{\mathrm{a}}$, Anamarija Knežević ${ }^{\mathrm{b}}$, Vladimir Vinković ${ }^{\mathrm{b}}$, Zrinka Kovarik ${ }^{\mathrm{a}}$, Goran Šinko ${ }^{\mathrm{a}, *}$ \\ a Institute for Medical Research and Occupational Health, Ksaverska cesta 2, HR-10 000 Zagreb, \\ Croatia \\ ${ }^{\mathrm{b}}$ Ruđer Bošković Institute, Bijenička cesta 54, HR-10 000 Zagreb, Croatia
}

\begin{abstract}
Within this study, we designed and synthesized four new oxime compounds of the $N$-substituted 2hydroxyiminoacetamide structure and evaluated their interactions with acetylcholinesterase (AChE) and butyrylcholinesterase (BChE). Our aim was to explore the possibility of extending the dual-binding mode of interaction between the enzyme and the inhibitor to a so-called triple-binding mode of interaction through the introduction of an additional binding moiety. $N$-substituted 2hydroxyiminoacetamide 1 was prepared via BOP catalyzed amidation of hydroxyiminoacetic acid with 3-azido-1-phenylpropylamine. An azide group enabled us to prepare more elaborate structures $\mathbf{2}-\mathbf{4}$ by the copper-catalyzed azide-alkyne cycloaddition. The new compounds $\mathbf{1}-\mathbf{4}$ differed in their presumed AChE peripheral site binding moiety, which ranged from an azide group to functionalized heterocycles. Molecular docking studies revealed that all three binding moieties are involved in the non-covalent interactions with ChEs for all of the four compounds, albeit not always in the complete accordance with the proposed hypothesis. All of the four compounds reversibly inhibited the ChEs with their inhibition potency increasing in the same order for both enzymes $(\mathbf{1}<\mathbf{2}<\mathbf{4}<\mathbf{3})$. A higher preference for binding to $\mathrm{BChE}\left(K_{\mathrm{i}}\right.$ from $0.30 \mu \mathrm{mol} / \mathrm{L}$ to $\left.130 \mu \mathrm{mol} / \mathrm{L}\right)$ over $\mathrm{AChE}\left(K_{\mathrm{i}}\right.$ from $50 \mu \mathrm{mol} / \mathrm{L}$ to 1200 $\mu \mathrm{mol} / \mathrm{L}$ ) was observed for all of the compounds.
\end{abstract}

Keywords: Oxime antidotes, Azide-alkyne cycloaddition, Organophosphorus compounds, Inhibition, Selectivity

* Corresponding author. Tel.: +38514682500

E-mail address: gsinko@imi.hr (G. Šinko) 


\section{Introduction}

Acetylcholinesterase (AChE; EC 3.1.1.7) is a key enzyme for the regulation of cholinergic transmission in both the central and peripheral nervous system that catalyzes the hydrolysis of the neurotransmitter acetylcholine (ACh) [1]. The decline of hippocampal and cortical levels of ACh is a characteristic of Alzheimer's disease (AD), a disabling and fatal neurodegenerative disease manifested by memory loss and learning deficits [2]. Thus, today's drugs designed for the treatment of AD are reversible AChE inhibitors that block the enzyme active site leading to an increase of ACh levels and, in turn, to the alleviation of disease symptoms [3].

The active site of $\mathrm{AChE}$ is a $20 \AA$ deep gorge divided into two sub-sites; the peripheral anionic site (PAS) (Tyr72, Tyr124, Trp286) located at the entrance of the gorge, and the catalytic site (CAS) located close to the bottom of the gorge. CAS is composed of the catalytic triad (Ser203, His447, Glu334), an oxyanion hole (Gly121, Gly122, Ala204), an acyl-binding pocket (Phe288, Phe290) and a choline binding site (Trp86, Tyr337, Phe338) [4,5]. The two sub-sites serve as the recognition sites for the ligands that bind to the AChE governing their mechanism of interaction. Depending on the established interactions, ligands can be described as PAS-binding or the CAS-binding. Out of several anti-AD drugs (Fig. 1), galanthamine (half-maximal inhibitory concentration $\left(\mathrm{IC}_{50}\right)$ of $2.01 \mu \mathrm{mol} / \mathrm{L}$ in human $\mathrm{AChE}$ ) [6,7] and huperzine $\mathrm{A}\left(\mathrm{IC}_{50}\right.$ of $0.082 \mu \mathrm{mol} / \mathrm{L}$ in mouse $\left.\mathrm{AChE}\right)[8,9]$ bind to the $\mathrm{CAS}$, while propidium iodide ( $\mathrm{IC}_{50}$ of $1.1 \mu \mathrm{mol} / \mathrm{L}$ in mouse $\mathrm{AChE}$ ) [10] binds in the PAS region [11]. Inhibitors that bind to PAS and CAS simultaneously include both symmetrical (e.g. bistacrine, $K_{\mathrm{d}}$ of $250 \mathrm{nmol} / \mathrm{L}$ in fetal bovine serum AChE) $[12,13]$ and non-symmetrical tacrine analogues (e.g. synTZ2PA6, $K_{\mathrm{d}}$ of $0.41 \mathrm{nmol} / \mathrm{L}$ in mouse AChE) [14,15], as well as donepezil $\left(K_{\mathrm{d}}\right.$ in hAChE of 3.35 $\mathrm{nmol} / \mathrm{L}$ ( $R$-donepezil), $17.5 \mathrm{nmol} / \mathrm{L}$ ( $S$-donepezil)) an anti-AD drug that binds with a basic nitrogen in the CAS and an aromatic system in the PAS [16, 17]. Crystal structures of AChE-inhibitor complexes have shown that inhibitors usually interact with gorge residues via arene-arene $(\pi-\pi)$ interactions. Trp86, which is essential for the interaction with the trimethylammonium group of $\mathrm{ACh}$, is considered crucial for the stabilization of CAS-binding ligands through cation- $\pi$ and $\pi-\pi$ interactions together with

the Tyr337, and His447 of the catalytic triad [18-20]. On the other hand, PAS-binding ligands are stabilized through $\pi-\pi$ interactions with Tyr72, Tyr124, and Trp286 [21-23].

The acute toxicity of organophosphorus (OP) nerve agents (e.g. tabun, soman, sarin, VX) is due to their irreversible inhibition of AChE by covalently binding to the catalytic serine residue which results in the accumulation of ACh in synaptic clefts [24]. The activity of AChE can be restored by 
treatment with a reactivator from the quaternary pyridinium oxime family (2-PAM, trimedoxime, obidoxime, HI-6, Hlö-7) (Fig. 1) by cleaving the covalent bond between the catalytic serine residue and the nerve agent [25,26]. HI-6 ( $K_{\mathrm{I}}$ for $\mathrm{hAChE}$ of $\left.20 \mu \mathrm{mol} / \mathrm{L}\right)$ [27] is an example of a quaternary bispyridinium oxime that binds to both the CAS and the PAS with its two positively charged heterocyclic aromatic rings [28]. The efficacy of both AChE inhibitors and reactivators currently used in medical treatment of $\mathrm{AD}$ or $\mathrm{OP}$ nerve agent poisoning is limited because they do not cross the blood-brain barrier readily due to their permanent positive charge [29].

Butyrylcholinesterase (BChE, E.C. 3.1.1.8) is related to AChE and it can also catalyze the hydrolysis of ACh; moreover, it serves as a co-regulator of cholinergic neurotransmission [30,31]. However, BChE plays an important role in the pathogenesis of $\mathrm{AD}$ with its activity increased at the early stage of disease and involvement in the amyloid $\beta$-peptide aggregation developing into senile plaque deposits [32,33]. The inhibition of BChE may thus be beneficial in the medical treatment of AD patients. AChE and BChE show a high resemblance with sequence homology of $65 \%[33,34]$. However, their active sites display different amino acids composition and therefore the BChE active site is about $200 \AA^{3}$ bigger [35,36], consequently allowing the BChE to bind and hydrolyze larger ligands and substrates than AChE [37]. Moreover, differences between AChE and BChE active site amino acid composition lead to $\mathrm{AChE} / \mathrm{BChE}$ selectivity for many ligands and substrates $[23,38]$. Some of the selective AChE inhibitors are BW284C51, huperzin A, and the aforementioned donepezil [8,17,24,23,]. Several selective BChE inhibitors have also been described, including bambuterol and ethopropazine (Fig. 1) [39-43]. Using ChE active site differences for designing selective inhibitors could help develop improved AD drugs and reactivators of OP nerve agent-inhibited enzymes.

In this study, we designed and synthesized four new compounds to probe the possibility of simultaneous non-covalent triple-binding between the inhibitors and the ChE. Our results could lead to the discovery of more selective ChE inhibitors as well as more effective reactivators of OP nerve agentinhibited enzymes. Compounds were designed by modifying the structures of $N$-substituted 2hydroxyiminoacetamides, recently introduced non-charged AChE oxime reactivators [44,45], through the introduction of a phenyl ring. It was expected that the phenyl ring would help their stabilization through $\pi-\pi$ interactions with active site aromatic amino acids and provide additional binding moiety apart from PAS- moiety and the 2-hidroxyiminoacetamide group. The working hypothesis was that the phenyl ring would interact with the choline binding site directing, together with PAS-binding moiety, the 2-hidroxyiminoacetamide group into a so-called third binding site surrounding Ser203. Molecular 
modelling was used to determine and visualize the binding modes of the new compounds and their interactions with the enzymes.

\section{Materials and methods}

\subsection{Chemicals}

$N$-substituted 2-hydroxyiminoacetamides $N$-(3-azido-1-phenylpropyl)-2-hydroxyiminoacetamide (1), $N$-(3-(4-cyclopentyl-1H-1,2,3-triazol-1-yl)-1-phenylpropyl)-2-hydroxyiminoacetamide (2), 2-hydroxyimino- $N$-(3-(4-((2-methyl-1 $H$-imidazol-1-yl)methyl)-1 $H$-1,2,3-triazol-1-yl)-1-phenylpropyl)acetamide (3), and 2-hydroxyimino- $N$-(3-(4-((2-hydroxyiminomethyl)-1 $H$-imidazol-1-yl)methyl)-1 $H$-1,2,3triazol-1-yl)-1-phenylpropyl)acetamide (4) were synthesized. 1 was prepared via BOP catalyzed amidation of hydroxyiminoacetic acid with 3-azido-1-phenylpropylamine [46]. 2 -4 were prepared by copper catalyzed azide-alkyne cycloaddition starting from 1 and a corresponding alkyne: ethynylcyclopentane, 2-methyl-1-(prop-2-yn-1-yl)-1H-imidazole, and 1-(prop-2-yn-1-yl)-1Himidazole-2-carbaldehyde oxime, respectively [47,48]. For more detailed information about the synthesis of 1-4, please refer to the Supplementary material.

\subsection{In vitro enzyme inhibition studies}

Reversible inhibition of $\mathrm{AChE/BChE}$ by $N$-substituted 2-hydroxyiminoacetamides was evaluated by determining the decrease of enzyme activity in the presence of $N$-substituted 2hydroxyiminoacetamides and substrate acetylthiocholine (ATCh). Enzyme activity was measured spectrophotometrically using the Ellman assay with 5,5'-dithiobis(2-nitrobenzoic acid) (DTNB) and substrate acetylthiocholine (ATCh, 0.1-0.3 mmol/L) [49]. Horse serum BChE was purchased from Sigma Chemical Co., USA. Final BChE dilution was 600 fold. Human recombinant AChE was prepared at the Jean-Pierre Ebel Institute of Structural Biology (IBS)-DYNAMOP, Grenoble, RhôneAlpes, France and was a gift from Dr. Florian Nachon. ATCh and DTNB were purchased from Sigma Chemical Co., USA. $N$-substituted 2-hydroxyiminoacetamides were dissolved in DMSO. ATCh and DTNB were dissolved in $0.1 \mathrm{~mol} / \mathrm{L}$ sodium phosphate buffer $(\mathrm{pH} 7.4)$. The reaction mixture contained the enzyme suspended in $0.1 \mathrm{~mol} / \mathrm{L}$ sodium phosphate buffer $(\mathrm{pH} 7.4), 0.3 \mathrm{mmol} / \mathrm{L} \mathrm{DTNB}, N$ substituted 2-hydroxyiminoacetamide and ATCh $(0.1-0.8 \mathrm{mmol} / \mathrm{L})$. The final assay volume was 300 $\mu \mathrm{L}$ and the enzymatic reaction was followed during 240 seconds at a temperature of $25{ }^{\circ} \mathrm{C}$ using a Tecan Infinite M200PRO plate reader (Tecan Group Ltd., Switzerland). To limit the influence of 
DMSO on the degree of enzyme inhibition, the final content of DMSO was held constant whenever it exceeded $0.05 \%$.

The inhibition constants were evaluated by the Enzyme Kinetics module of Graph Pad Prism version 6.01 (GraphPad Software, Inc., USA). The dose response curves were fitted using the Mixed Model Inhibition equation:

$$
\begin{aligned}
& v_{\mathrm{i}}=\frac{V_{\mathrm{m}}^{\prime} \cdot \mathrm{S}}{K_{\mathrm{m}}^{\prime}+\mathrm{S}} \\
& V_{\mathrm{m}}^{\prime}=V_{\mathrm{m}} \cdot \frac{1}{\left(1+\frac{\mathrm{I}}{\alpha K_{\mathrm{I}}}\right)} \quad K_{\mathrm{m}}^{\prime}=K_{\mathrm{m}} \cdot \frac{\left(1+\frac{\mathrm{I}}{K_{\mathrm{I}}}\right)}{\left(1+\frac{\mathrm{I}}{\alpha K_{\mathrm{I}}}\right)}
\end{aligned}
$$

where $\mathrm{S}$ is the concentration of substrate ATCh, I is the concentration of inhibitor (oxime), $K_{\mathrm{I}}$ is the enzyme-oxime inhibition (dissociation) constant of a complex formed at the catalytic site, $\alpha K_{\mathrm{I}}$ is the Michaelis complex-oxime inhibition (dissociation) constant of a complex formed at the peripheral site, $K_{\mathrm{m}}$ is a dissociation constant of the Michaelis complex, and $V_{\mathrm{m}}$ is maximal activity.

\subsection{Molecular modelling}

Compounds to be docked in the active site of human AChE and human BChE were created and minimized using the MMFF94 force field implemented in ChemBio3D Ultra 12.0 (PerkinElmer, Inc., USA).

Accelrys Discovery Studio's Dock Ligands protocol (CDOCKER) was used for the docking study with CHARMm force field (Accelrys, USA) [50,51]. The crystal structure of human AChE (PDB: 1B41, 4PQE) [52] or human BChE (PDB: 2PM8) [53] was used as the rigid receptor. The binding site within the $\mathrm{AChE}$ or $\mathrm{BChE}$ was defined as the largest cavity in the enzyme structure surrounded by a sphere $(r=13 \AA)$. The following steps were included in the CDOCKER protocol. First, a set of 20 random ligand conformations for each test compound was generated. In the following step, 20 random orientations were kept if the energy was less than the specified threshold value of 300 $\mathrm{vdW}$. This process continued until either a desired number of low-energy orientations were found or the maximum number of bad orientations had been attempted. The maximum number of bad orientations was set to 800. In the next step each orientation was subjected to simulated annealing molecular dynamics. The temperature was increased to $700 \mathrm{~K}$ then cooled to $310 \mathrm{~K}$. The numbers of heating and cooling phase steps during simulated annealing were set to 2000 and 5000, respectively. For the simulated annealing refinement, grid extension $(8.0 \AA)$ was used. In the subsequent step, a final 
minimization of each refined pose of the ligand in the rigid receptor is performed using full potential. In the end, for each final pose, the CHARMm energy (interaction energy plus ligand strain) and the interaction energy alone are calculated. The poses are sorted by CHARMm energy and the 20 top scored (most negative, thus favorable for binding) poses are retained.

The selected poses for each enzyme-ligand complex were minimized using protocol Minimization with Smart Minimizer algorithm. The applied algorithm performs 1000 steps of Steepest Decent with a RMS gradient tolerance of 3, followed by Conjugate Gradient minimization with the values of Max Steps and RMS Gradient set to 500 and 0.01, respectively. Generalized Born with Molecular Volume implicit solvent model was used [54,55]. The non-polar surface area was used to approximate the non-polar component of the solvation energy. Implicit solvent dielectric constant was set to 80. Distance cutoff value used for counting non-bonded interaction pairs was set to $14.0 \AA$.

\section{Results and discussion}

\subsection{Kinetic measurements}

Based on the hypothesis that the dual-binding mode of interaction between $\mathrm{AChE} / \mathrm{BChE}$ and its inhibitors or reactivators can be extended to a so-called triple-binding mode of interaction, we designed and synthesized four new compounds capable of simultaneous non-covalent triple-binding with enzymes. In doing so, we modified the structures of recently reported $N$-substituted 2hidroxyimnoacetamides [44] by introducing the phenyl ring. Some of the reported $N$-substituted 2hidroxyiminoacetamides are known to possess high reactivation potential toward sarin-, cyclosarin-, and VX-inhibited AChE [45]. According to our hypothesis, the phenyl ring was expected to bind in the choline binding site. The presumed PAS-binding moieties ranged from an azide group to functionalized heterocycles and were connected with the central $N$-(1-phenylpropyl)-2-hydroxyiminoacetamide scaffold via a 1,2,3-triazole ring. Following $N$-substituted 2-hydroxyiminoacetamides were synthesized: $N$-(3-azido-1-phenylpropyl)-2-hydroxyiminoacetamide (1), $N$-(3-(4-cyclopentyl-1H-1,2,3triazol-1-yl)-1-phenylpropyl)-2-hydroxyiminoacetamide (2), 2-hydroxyimino- $N$-(3-(4-((2-methyl-1Himidazol-1-yl)methyl)-1H-1,2,3-triazol-1-yl)-1-phenylpropyl)acetamide (3), and 2-hydroxyimino- $N$-(3(4-((2- hydroxyiminomethyl)-1H-imidazol-1-yl)methyl)-1H-1,2,3-triazol-1-yl)-1-phenylpropyl)acetamide (4) (Fig. 2).

To determine the binding affinity of $\mathrm{AChE}$ and $\mathrm{BChE}$ for $\mathbf{1} \mathbf{- 4}$, we performed detailed enzyme kinetics measurements (Fig. 3). All four of the $N$-substituted 2-hydroxyiminoacetamides reversibly inhibited both $\mathrm{AChE}$ and $\mathrm{BChE}$ displaying mixed types of inhibition. This suggests that all four oximes 
can bind to the free enzyme (E) and to the Michaelis complex (ES). Parameter $\alpha>1$ describes the decrease in the ES complex affinity for an oxime in comparison to the affinity of the free enzyme (E). All of the tested oximes bind more weakly to the ES than to the E in the case of AChE, except for 2. However, due to the low solubility of 2 in $0.1 \mathrm{M}$ sodium phosphate buffer, its kinetic parameters could not be evaluated at the optimal concentration range which may explain its exceptional behavior. For $\mathrm{BChE}$, this decrease in ES affinity is even more pronounced indicating obstruction of interactions between the oxime and gorge residues due to the substrate presence. Inhibition constants $\left(K_{\mathrm{i}}\right)$ for $\mathrm{AChE}$ ranged from 50 to $1200 \mu \mathrm{mol} / \mathrm{L}$ (Table 1) with the inhibition potency increasing in the following order: $1<2<4<3$. For BChE, $K_{\mathrm{i}}$ ranged from 0.3 to $130 \mu \mathrm{mol} / \mathrm{L}$ with the same order of inhibition potency as the one observed for AChE. Results show that our modification of the presumed PAS-binding moieties can influence inhibition potency significantly. Compound $\mathbf{1}$ displayed the lowest affinity toward the enzymes, which is probably the result of the lack of a more elaborated structure of its presumed PAS-binding moiety, i.e. an azide group present in 2, 3 and 4. On the other hand, 3 proved to be the most potent inhibitor of both enzymes. Also, all four oximes demonstrated a preference for binding to BChE, probably due to a bigger BChE active site compared to AChE allowing such bulkier ligands to adopt more favorable binding conformation [37]. Also, 6 out of 14 aromatic amino acids in the AChE active site corresponding to aliphatic ones in the BChE site made it more hydrophobic and favorable for lipophilic compounds. Moreover, 3 displayed an almost 150 times higher affinity for BChE compared to $\mathrm{AChE}$, thus $\mathbf{3}$ can be considered a selective BChE inhibitor.

\subsection{Molecular modeling}

In order to reveal the key interactions leading to the observed differences in binding affinity of 1-4 and their preference for binding to $\mathrm{BChE}$, molecular docking studies were conducted using structures of human AChE (PDB: 1B41, 4PQE) and human BChE (PDB: 2PM8) (Fig. 4 and 5). 1-4 were docked into the active site of the enzyme. The resulting poses were critically investigated targeting the ones including $\pi-\pi$ interactions between a compound and aromatic amino acids of PAS and choline binding sites - a type of interaction typical of enzyme-inhibitor/reactivator complex observed by X-ray crystallography for both $\mathrm{AChE}$ and $\mathrm{BChE}$ [12,18-23,28,56,57]. Poses that had fulfilled these criteria were chosen for the prediction of key interactions between the compound and the enzyme summarized in Table 2. In agreement with experimentally determined enzyme- $N$-substituted 2hydroxyiminoacetamide inhibition constants, 3 displayed the highest number of predicted interactions with both enzymes, while the weakest inhibitor 1 displayed the lowest number of interactions. On the 
other, it was clear that the differences in inhibition potencies and their preference for binding to BChE could not be attributed solely to the number of interactions with the enzyme.

\subsubsection{Modeling of AChE complexes}

The predicted binding geometry of $\mathbf{1}$ in the AChE active site supported our hypothesis that a phenyl ring would bind in the choline binding site yielding $\pi-\pi$ interactions with Trp86 (Fig. 4A). As mentioned, this type of interaction has been confirmed from structures for numerous complexes of ChEs and aromatic ring-containing ligands, i.e. parallel $\pi-\pi$ stacking between the benzyl ring of the donepezil complexed with human (h) AChE Trp86 or Torpedo californica (Tc) AChE Trp84 [17,58], $\pi-\pi$ stacking of the tacrine ring against the Trp84 in the tacrine-TcAChE complex [59] and bistacrineTcAChE complex [13], and against the Trp82 in the tacrine-hBChE complex [56]. Also in accordance with our hypothesis, the 2-hydroxyiminoacetamide moiety was directed toward the catalytic serine Ser203 making hydrogen bonds between its hydroxyl group and Ser203 and/or His447 of the catalytic triad. Additionally, a side chain of $\mathbf{1}$ with an azide group makes hydrogen bonds with Tyr133. This residue makes hydrogen bonds with ligands, i.e. (-)-huperzine A in complex with hAChE [56]. The lack of interactions between 1 and the residues in the PAS region could explain its low inhibition potential when compared to other $N$-substituted 2-hydroxyiminoacetamides.

Model complex of 2 and AChE (Fig. 4B) also predicts $\pi$ - $\pi$ interactions between the phenyl ring and Trp86. The triazole ring, absent in 1, makes hydrogen bonds with Tyr124 which is similar to a hydrogen bond between the phenol ring of Tyr124 and the pyridinium ring of the 2-hydroxyiminomethylpyridinium ring of HI-6 in complex with mAChE [60]. These interactions seem to govern the overall binding mode of $\mathbf{2}$ in the AChE active site directing the presumed PAS-binding moiety, i.e. the cyclopentyl ring, in the PAS and the 2-hydroxyiminoacetamide moiety toward the catalytic serine with which it makes hydrogen bonds via its oxime group [57].

Elongated binding conformation of $\mathbf{3}$ in the AChE active site (Fig. 4C) is characterized with a methylimidazole ring, directed towards the entry of the AChE gorge and the 2-hydroxyiminoacetamide moiety directed towards the bottom of the gorge. The geometry of $\mathbf{3}$ in the AChE gorge leads to multiple $\pi-\pi$ interactions with aromatic amino acids; the imidazole ring with $\operatorname{Trp} 286$, the triazole ring with Tyr341 and with Phe297 ( $\pi$-sigma interaction), and the phenyl ring interacts with Tyr124. This is in accordance with X-ray structures of oximes HI-6, Ortho-7, and obidoxime in complex with $m$ AChE [58], and donepezil in complex with TcAChE [17]. Additionally, the 2-hydroxyiminoacetamide moiety of $\mathbf{3}$ creates a hydrogen bond with Glu202. 
The predicted binding geometry of $\mathbf{4}$ in the AChE (Fig. 4D) suggests that $\mathbf{4}$ also binds with its presumed PAS-binding moiety, imidazole-2-carboxaldehyde oxime, at the entry of the gorge and the phenyl ring at the choline binding site. The triazole ring binds in the narrow part defined by Tyr124 and Tyr337. The only difference between the predicted binding geometry of $\mathbf{3}$ and $\mathbf{4}$ was that $\mathbf{4}$ had been buried deeper in the $\mathrm{AChE}$ active site. This results in $\pi-\pi$ interactions between the imidazole-2carboxaldehyde oxime and Trp341. Additionally, 2-hydroxyiminoacetamide group makes hydrogen bonds with Glu202 and Ser203. However, this also leads to the loss of interaction between the imidazole ring and PAS residues Tyr124 and Trp286 which could explain the lower inhibition potential of $\mathbf{4}$ when compared to $\mathbf{3}$.

\subsubsection{Modeling of BChE complexes}

The most commonly observed change in the predicted binding geometry of $\mathbf{1 - 4}$ in the BChE active site when compared to those for AChE has to do with the energetically more favorable bended conformations of oximes in the $\mathrm{BChE}$ active site, a finding which reflects the larger $\mathrm{BChE}$ active site volume. The complex of $\mathbf{1}$ in the BChE active site (Fig. 5A) again supports our hypothesis that a phenyl ring would bind in the choline binding site making $\pi-\pi$ interactions with $\operatorname{Trp} 82$. However, in the $\mathrm{BChE}$ active site the phenyl ring is placed closer to the center of the choline binding site. This change in the positioning of the phenyl ring is made possible because bulky Tyr337 in the AChE corresponds to smaller Ala328 in the BChE. Additionally, the side chain modified with an azide group is oriented almost parallel to the Trp82 main chain making $\pi-\pi$ interactions with its indole ring and the 2hydroxyiminoacetamide moiety is directed towards Ser203 making multiple hydrogen bonds. Once again, the lack of interactions between $\mathbf{1}$ and the residues in the PAS region could explain its lowest inhibition potential among all of the tested compounds.

The complex of 2 and BChE (Fig. 5B) predicts binding for 2 almost perpendicularly to the axis connecting the entry and the bottom of the active site gorge. This orientation is made possible because aromatic Tyr124, Phe297, and Tyr337 in the AChE active site correspond to smaller Gln119, Val288, and Ala328 in the BChE active site, respectively. Otherwise, these aromatic amino acids in the AChE active site would not allow such an orientation of $\mathbf{2}$. Thereby, the cyclopentyl ring occupies the space normally inaccessible in the AChE active site. 2-hydroxyiminoacetamide moiety is directed towards the acyl pocket with its hydroxyl group and making hydrogen bonds with Leu286. Additionally, the carbonyl oxygen of the 2-hydroxyiminoacetamide moiety makes hydrogen bonds with Ser198. Contrary to our hypothesis, the phenyl ring is shifted towards the entry of the BChE active site gorge, 
but is just close enough to Phe329 to make a $\pi-\pi$ interaction. This distinct residue corresponds to Phe338 in the AChE active site which participates in aromatic interactions, the donepezil complex with TcAChE [17] and the HI-6 complex with $m$ AChE [60].

Model complex of $\mathbf{3}$ and BChE (Fig. 5C) predicts geometry where $\mathbf{3}$ is in a bended conformation and is placed in the center of the BChE active site. Once again this is expected due to Gln119, Val288, and Ala328. Thereby, the methylimidazole ring occupies the volume otherwise restricted in the AChE active site because of the Tyr337 side chain and interacts with Trp82 and His438 via $\pi-\pi$ interactions. The 2-hydroxyiminoacetamide moiety is directed towards the bottom of the active site where its amide hydrogen makes hydrogen bonds with the backbone of Pro285 and the oxime group makes hydrogen bonds with Gly116 and Gly117 from an oxyanion hole, as well as with Ser198. It has been assumed that hydrogen bonding with Pro285 has been partially responsible for the inhibition differences of horse, human, and mouse BChE [41].

The complex of 4 and BChE (Fig. 5D) shows that the imidazole-2-carboxaldehyde oxime and the following triazole ring are located in the upper part of the active site while the phenyl ring and the 2-hydroxyiminoacetamide moiety are located close to the bottom of the active site. Such positioning of the triazole ring allows it to make $\pi-\pi$ interactions with Tyr332 that corresponds to Tyr341 in the AChE site. In support of our hypothesis, the phenyl ring was placed at the bottom of the BChE active site close enough to yield a $\pi-\pi$ interaction with Trp82. This distinct tryptophan residue is also involved in $\pi$-sigma interaction with a hydrogen atom from one of the 4 methylene groups and Trp82 main chain makes a hydrogen bond with the hydroxyl group of the 2-hydroxyiminoacetamide moiety. The former interaction could be related to that between the methylene group in the tetrahydroazepine ring of (-)galanthamine in complex with $T c \mathrm{AChE}$ [57]. The 2-hydroxyiminoacetamide moiety is located close to the center of the $\mathrm{BChE}$ active site and is almost perpendicular to the axis connecting the entry and the bottom of the active site gorge. Also, it is directed into the area between the Trp82 main chain and Thr120 side chain with which it makes another hydrogen bond via its carbonyl oxygen.

\section{Conclusion}

All four compounds reversibly inhibited BChE with inhibition constants ranging from 0.30 $\mu \mathrm{mol} / \mathrm{L}$ to $130 \mu \mathrm{mol} / \mathrm{L}$. The inhibition potency of compounds increased in the following order: $1<2<$ $4<3$. AChE was also reversibly inhibited by all compounds with the same order of inhibition potency. Inhibition constants ranged from $50 \mu \mathrm{mol} / \mathrm{L}$ to $1200 \mu \mathrm{mol} / \mathrm{L}$. All of the compounds displayed a higher preference for binding to $\mathrm{BChE}$. 
It can be concluded from molecular docking studies that the predicted binding modes of $\mathbf{2}-\mathbf{4}$ in the AChE active site support our hypothesis of a so-called triple-binding mode of interaction with the enzyme, with the presumed PAS-binding moiety in the PAS, the phenyl ring in the choline binding site, and the 2-hydroxyiminoacetamide moiety in the third binding site surrounding active serine. On the other hand, none of the $N$-substituted 2-hydroxyiminoacetamides is predicted to bind in the $\mathrm{BChE}$ active site in complete accordance with the above stated hypothesis, though all three binding groups are involved in non-covalent interactions with the enzyme. However, most of the predicted interactions between $N$-substituted 2-hydroxyiminoacetamides and ChEs could be supported with interactions observed from the X-ray structures of various ligands complexed with ChE. To the best of our knowledge, only a few interactions in the BChE active site, namely $\pi-\pi$ interactions between His 438 and the methylimidazole ring of $\mathbf{3}$, hydrogen bond between Phe295 and the 2-hydroxyiminoacetamide moiety of $\mathbf{3}$, hydrogen bond between Trp82 and the 2-hydroxyiminoacetamide moiety of $\mathbf{4}$, and the hydrogen bond between Thr120 and the 2-hydroxyiminoacetamide moiety of 4 could not have been supported with interactions revealed by X-ray crystallography. The differences in the predicted binding modes of 1-4 between the two enzymes reflect different stereoelectronic properties of their active sites caused because 6 out of 14 aromatic amino acids in the AChE active site corresponded to aliphatic ones in the BChE active site. The most important ones that primarily governed the differences in the predicted binding modes of $\mathbf{1 - 4}$ occured at the position of Tyr72, Tyr124, Phe297 and Tyr337 in the AChE active site.

Knowledge of the degree of $\mathrm{AChE} / \mathrm{BChE}$ selectivity of oxime compounds is may be important for more successful treatment in cases of OP nerve agent poisoning. The most notable pretreatment strategies include protection of the AChE catalytic serine from phosphylating agent by ligands that bind reversibly to $\mathrm{AChE} \mathrm{[61-65]} \mathrm{and} \mathrm{the} \mathrm{use} \mathrm{of} \mathrm{bioscavengers,} \mathrm{i.e.} \mathrm{BChE,} \mathrm{prone} \mathrm{to} \mathrm{inhibition} \mathrm{by} \mathrm{a}$ phosphylating agent $[66,67]$. Furthermore, BChE is considered to act as a natural bioscavenger in the bloodstream [68,69]. Considering the especially high degree of preference for binding to BChE displayed by $N$-substituted 2-hydroxyiminoacetamide $\mathbf{3}$, our results clearly discourage its use in the protection of the AChE catalytic site from a phosphylating agent by reversible inhibition of AChE. Moreover, it would also diminish the BChE endogenous bioscavenging capability by inhibiting BChE if administered prior to the OP nerve agent exposure. However, if its preference for binding to BChE proved to be retained in the case of a phosphylated enzyme and was followed by a fast reactivation, together with BChE it could make an enzyme-oxime pair acting as a pseudo catalytic-scavenger [70]. 


\section{Conflict of Interest}

The authors declare that there are no conflicts of interest associated with this work.

\section{Acknowledgements}

The authors thank Dr. Florian Nachon for recombinant human AChE. This study was supported by the Croatian Science Foundation (Grant 4307).

\section{References}

[1] J.L. Taylor, R.T. Mayer, C.M. Himel, Conformers of acetylcholinesterase - a mechanism of allosteric control, Mol. Pharm. 45 (1994) 74-83.

[2] E.K. Perry, B.E. Tomlinson, G. Blessed, K. Bergmann, P.H. Gibson, R.H. Perry, Correlation of cholinergic abnormalities with senile plaques and mental test scores in senile dementia, Br. Med. J. 2 (1978) 1457-1459.

[3] A. Enz, R. Amstutz, H. Boddeke, G. Gmelin, J. Malonowski, Brain selective inhibition of acetylcholinesterase: a novel approach to therapy for Alzheimer's disease, Prog. Brain Res. 98 (1993) 431-435.

[4] P. Taylor, Z. Radić, The cholinesterases: from genes to proteins, Annu. Rev. Pharmacol. Toxicol. 34 (1994) 281-320.

[5] A. Ordentlich, D. Barak, C. Kronman, Y. Flashner, M. Leitner, Y. Segall, N. Ariel, S. Cohen, B. Velan, A. Shafferman, Dissection of the human acetylcholinesterase active centre determinants of substrate specificity. Identification of residues constituting the anionic site, the hydrophobic site, and the acyl pocket, J Biol Chem 268 (1993) 17083-17095.

[6] M.F. Eskander, N.G. Nagykery, E.Y. Leung, B. Khelghati, C. Geula, Rivastigmine is a potent inhibitor of acetyl- and butyrylcholinesterase in Alzheimer's plaques and tangles, Brain Res. 1060 (2005) 144-152.

[7] C. Galdeano, E. Viayna, P. Arroyo, A. Bidon-Chanal, J.R. Blas, D. Munoz-Torrero, F.J. Luque, Structural determinants of the multifunctional profile of dual binding site acetylcholinesterase inhibitors as anti-Alzheimer agents, Curr. Pharm. Des. 16 (2010) 2818-2836.

[8] R.W. Zhang, X.C. Tang, Y.Y. Han, G.W. Sang, Y.D. Zhang, Y.X. Ma, C.L. Zhang, R.M. Yang, Drug-evaluation of huperzine-a in the treatment of senile memory disorders, Acta Pharm. Sin. 12 (1991) 250-252.

[9] R. Wang, H. Yan, X.C. Tang, Progress in studies of huperzine A, a natural cholinesterase inhibitor from Chinese herbal medicine, Acta Pharmacol. Sin. 27 (2006) 1-26.

[10] Z. Radić, P. Taylor, Interaction kinetics of reversible inhibitors and substrates with acetylcholinesterase and its fasciculin 2 complex, J. Biol. Chem. 276 (2001) 4622-4633.

[11] Y. Bourne, P. Taylor, Z. Radić, P. Marchot, Structural insights into ligand interactions at the acetylcholinesterase peripheral anionic site, Embo J. 22 (2003) 1-12.

[12] P.R. Carlier, Y.F. Han, E.S.H. Chow, C.P.L. Li, H. Wang, T.X. Lieu, H.S. Wong, Y.P. Pang, Evaluation of short-tether bis-THA AChE inhibitors. A further test of the dual binding site hypothesis, Bioorg. Med. Chem. 7 (1999) 351-357. 
[13] E.H. Rydberg, B. Brumshtein, H.M. Greenblatt, D.M. Wong, D.Shaya, L.D. Williams, P.R. Carlier, Y.P. Pang, I. Silman, J.L. Sussman, Complexes of alkylene-linked tacrine dimers with Torpedo californica acetylcholinesterase: binding of bis(5)-tacrine produces a dramatic rearrangement in the active-site gorge, J. Med. Chem. 49 (2006) 5491-5500.

[14] W.G. Lewis, L.G. Green, F. Grynszpan, Z. Radić, P.R. Carlier, P.Taylor, M.G. Finn, K.B. Sharpless, Click chemistry in situ: acetylcholinesterase as a reaction vessel for the selective assembly of a femtomolar inhibitor from an array of building blocks, Angew. Chem. Int. Ed. 41 (2002) 1053-1057.

[15] Y. Bourne, H.C. Kolb, Z. Radić, K.B. Sharpless, P. Taylor, P. Marchot, Freeze-frame inhibitor captures acetylcholinesterase in a unique conformation, P. Natl. Acad. Sci. USA 101 (2004) 1449-1454.

[16] A. Inoue, T. Kawai, M. Wakita, Y. Imura, H. Sugimoto, Y. Kawakami, The simulated binding of (+/-)-2,3-dihydro-5,6-dimethoxy-2-[(1-(phenylmethyl)-4-piperidinyl)methyl]-1H-inden-1-one

hydrochloride (E2020) and related inhibitors to free and acylated acetylcholinesterases and corresponding structure-activity analyses, J. Med. Chem. 39 (1996) 4460-4470.

[17] G. Kryger, I. Silman, J.L. Sussman, Structure of acetylcholinesterase complexed with E2020 (Aricept (R)): implications for the design of new anti-Alzheimer drugs, Structure 7 (1999) 297-307.

[18] D.A. Dougherty, D.A. Stauffer, Acetylcholine binding by a synthetic receptor: implications for biological recognition, Science 250 (1990) 1558-1560.

[19] D.A. Dougherty, Cation-pi interactions in chemistry and biology: a new view of benzene, Phe, Tyr, and Trp, Science 271 (1996) 163-168.

[20] I. Silman, M. Harel, J. Eichler, J.L. Sussman, A. Anselmet, J. Massoulié, Structure-Function Relationships in The Binding of Reversible Inhibitors in the Active-Site Gorge of Acetylcholinesterase, in: R. Becker, E. Giacobini (Eds.), Alzheimer Disease: Therepeutic Strategies. Birkäuser, Boston, 1994, pp. 88-92.

[21] Z. Radić, R. Durán, D.C. Vellom, Y. Li, C. Cerveñansky, P. Taylor, Site of fasciculin interaction with acetylcholinesterase, J. Biol. Chem. 269 (1994) 11233-11239.

[22] Y. Bourne, P. Taylor, P. Marchot, Acetylcholinesterase inhibition by fasciculin: crystal structure of the complex. Cell 83 (1995) 503-512.

[23] Z. Radić, N.A. Pickering, D.C. Vellom, S. Camp, P. Taylor, Three distinct domains in the cholinesterase molecule confer selectivity for acetyl- and butyrylcholinesterase inhibitors, Biochemistry 32 (1993) 12074-12084.

[24] S.M. Somani, J.A. Romano Jr., Chemical Warfare Agents: Toxicity at Low Levels. CRC Press LLC, Boca Raton, Florida 2001.

[25] M.P. Stojiljković, M. Jokanović, Pyridinium oximes: Rationale for their selection as causal antidotes against organophosphate poisonings and current solutions for auto-injectors, Arh. Hig. Rada Toksikol. 57 (2006) 435-443.

[26] F. Worek, P. Eyer, N. Aurbek, L. Szinicz, H. Thiermann, Recent advances in evaluation of oxime efficacy in nerve agent poisoning by in vitro analysis, Toxicol. Appl. Pharmacol. 219 (2007) 226-234. [27] G. Šinko, J. Brglez, Z. Kovarik, Interactions of pyridinium oximes with acetylcholinesterase, Chem. Biol. Interact. 187 (2010) 172-176. 
[28] F. Ekström, A. Hörnberg, E. Artursson, L.G. Hammarström, G. Schneider, Y.P. Pang, Structure of HI-6-sarin-acetylcholinesterase determined by X-ray crystallography and molecular dynamics simulation: Reactivator mechanism and design, PLoS One 4 (2009) e5957, doi:10.1371/journal.pone.0005957.

[29] P. Taylor, Anticholinesterase agents, in: L.L. Brunton, B.A. Chabner, B.C. Knollman (Eds.), Goodman and Gilman's the Pharmacological Basis of Therapeutics. McGraw-Hill, New York, 2011, pp. 239-254.

[30] V. Kumar, Introduction to cholinesterase inhibitors used in Alzheimer's disease therapy, in: R. Becker, E. Giacobini (Eds.), Alzheimer Disease: Therepeutic Strategies. Birkäuser, Boston, 1994, pp. 99-102.

[31] M.M. Mesulam, A. Guillozet, P. Shaw, A. Levey, E.G. Duysen, O. Lockridge, Acetylcholinesterase knockouts establish central cholinergic pathways and can use butyrylcholinesterase to hydrolyze acetylcholine, Neuroscience 110 (2002) 627-639.

[32] S. Darvesh, D.A. Hopkins, C. Geula, Nat. Neurobiology of butyrylcholinesterase. Neurosci. 4 (2003) 131-138.

[33] V.N. Talesa, Acetylcholinesterase in Alzheimer's disease, Mech. Ageing Dev. 122 (2001) 19611969.

[34] Y. Nicolet, O. Lockridge, P. Masson, J.C. Fontecilla-Camps, F. Nachon, Crystal structure of human butyrylcholinesterase and of its complexes with substrate and products, J. Biol. Chem. 278 (2003) 41141-41147.

[35] H. Dvir, I. Silman, M. Harel, T.L. Rosenberry, J.L. Sussman, Acetylcholinesterase: From 3D Structure to Function. Chem. Biol. Interact. 187 (2010) 10-22.

[36] N.A. Çokuğras, Butyrylcholinesterase: structure and physiological importance. Turk. J. Biochem. 28 (2003) 54-61.

[37] A. Saxena, A.M.G. Redman, X. Jiang, O. Lockridge, B.P Doctor, Differences in active-site gorge dimensions of cholinesterase revealed by binding of inhibitors to human butyrylcholinesterase, Chem. Biol. Interact 119-120 (1999) 61-69.

[38] P. Taylor, Z. Radić, N.A. Hosea, S. Camp, P. Marchot, H.A. Berman, Structural bases for the specificity of cholinesterase catalysis and inhibition, Toxicol. Lett. 82-83 (1995) 453-458.

[39] Z. Kovarik, Z. Radić, B. Grgas, M. Škrinjarić-Špoljar, E. Reiner, V. Simeon-Rudolf, V,

Amino acid residues involved in the interaction of acetylcholinesterase and butyrylcholinesterase with the carbamates Ro 02-0683 and bambuterol, and with terbutaline, Biochim. et Biophys. Acta 1433 (1999) 261-271.

[40] Z. Kovarik, V. Simeon-Rudolf, Interaction of human butyrylcholinesterase variants with bambuterol and terbutaline, J. Enzym. Inhib. Med. Chem. 19 (2004) 113-117.

[41] Z. Kovarik, A. Bosak, G. Šinko, T. Latas, Exploring active sites of cholinesterases by inhibition with bambuterol and haloxon. Croat. Chem. Acta 76 (2003) 63-67.

[42] M. Goličnik, G. Šinko, V. Simeon-Rudolf, Z. Grubič, J. Stojan, Kinetic model of ethopropazine interaction with horse serum butyrylcholinesterase and its docking into the active site, Arch. Biochem. Biophys. 398 (2002) 23-31. 
[43] G. Šinko, Z. Kovarik, E. Reiner, V. Simeon-Rudolf, J. Stojan, Mechanism of stereoselective interaction between butyrylcholinesterase and ethopropazine enantiomers, Biochimie 93 (2011) 1797-1807.

[44] R.K. Sit, Z. Radić, V. Gerardi, L. Zhang, E. Garcia, M. Katalinić, G. Amitai, Z. Kovarik, V.V. Fokin, K.B. Sharpless, P. Taylor, New structural scaffolds for centrally acting oxime reactivators of phosphylated cholinesterases, J. Biol. Chem. 286 (2011) 19422-19430.

[45] Z. Kovarik, N. Maček, R.K. Sit, Z. Radić, V.V. Fokin, K.B. Sharpless, P. Taylor, Centrally acting oximes in reactivation of tabun-phosphoramidated AChE, Chem. Biol. Interact. 203 (2013) 77-80.

[46] A. Knežević, V. Vinković, N. Maraković, G. Šinko, Enzyme-catalysed cascade synthesis of hydroxyiminoacetamides, Tetrahedron Lett. 55 (2014) 4338-4341.

[47] V.V. Rostovtsev, L.G. Green, V.V. Fokin, K.B. Sharpless, A stepwise huisgen cycloaddition process: copper(I)-catalyzed regioselective "ligation" of azides and terminal alkynes, Angew. Chem., Int. Ed. 41 (2002) 2596-2599.

[48] P.K. Avti, D. Maysinger, A. Kakkar, Alkyne-Azide "Click" Chemistry in Designing Nanocarriers for Applications in Biology, Molecules 18 (2013) 9531-9549.

[49] G.L. Ellman, K.D. Courtney, V. Andres, R.M. Featherstone, A new and rapid colorimetric determination of acetylcholinesterase activity, Biochem. Pharmacol. 7 (1961) 88-95.

[50] B.R. Brooks, R.E. Bruccoleri, B.D. Olafson, D.J. States, S. Swaminathan, M. Karplus, CHARMM: A program for macromolecular energy, minimization, and dynamics calculations, J. Comp. Chem. 4 (1983) 187-217.

[51] F.A. Momany, R. Rone, Validation of the general purpose QUANTA 3.2/CHARMm force field, J. Comp. Chem. 13 (1992) 888-900.

[52] G. Kryger, M. Harel, K. Giles, L. Toker, B. Velan, A. Lazar, C. Kronman, D. Barak, N. Ariel, A. Shafferman, I. Silman, J.L. Sussman. Structures of recombinant native and E202Q mutant human acetylcholinesterase complexed with the snake-venom toxin fasciculin-II, Acta Crystallogr., Sect. D 56 (2000) 1385-1394

[53] M.N. Ngamelue, K. Homma, O. Lockridge, O.A. Asojo, Crystallization and X-ray structure of full-length recombinant human butyrylcholinesterase, Acta Crystallogr., Sect. F 63 (2007) 723-727.

[54] M. Feig, C.L. Brooks III, Recent advances in the development and application of implicit solvent models in biomolecule simulations, Curr. Opin. Struct. Biol. 14 (2004) 217-224.

[55] M. Nina, D. Beglov, B. Roux, Atomic Born radii for continuum electrostatic calculations based on molecular dynamics free energy simulations, J. Phys. Chem. B. 101 (1997) 5239-5248.

[56] F. Nachon, E. Carletti, C. Ronco, M. Trovaslet, Y. Nicolet, L. Jean, P.-Y. Renard, Crystal structures of human cholinesterases in complex with huprine $\mathrm{W}$ and tacrine: elements of specificity for anti-Alzheimer's drugs targeting acetyl- and butyryl-cholinesterase, Biochem. J. 453 (2013) 393-399.

[57] H.M. Greenblatt, G. Kryger, T. Lewis, I. Silman, J.L. Sussman, Structure of acetylcholinesterase complexed with (-)-galanthamine at $2.3 \AA$ resolution, FEBS Lett. 463 (1999) 321-326.

[58] J. Cheung, M.J. Rudolph, F. Burshteyn, M.S. Cassidy, E.N. Gary, J. Love, M.C. Franklin, J.J. Height, Structures of human acetylcholinesterase in complex with pharmacologically important ligands, J. Med. Chem. 55 (2012) 10282-10286. 

J.L. Sussman, Quaternary ligand binding to aromatic residues in the active-site gorge of acetylcholinesterase, Proc. Natl. Acad. Sci USA 90 (1993) 9031-9035.

[60] F. Ekström, Y.-P. Pang, M. Boman, E. Artursson, C. Akfur, S. Börjegren, Crystal structures of acetylcholinesterase in complex with HI-6, Ortho-7 and obidoxime: Structural basis for differences in the ability to reactivate tabun conjugates, Biochem. Pharmacol. 72 (2006) 597-607.

[61] L.W. Harris, W.C. Heyl, D.L. Stitcher, C.A. Broomfield, Effects of 1.1'-oxydimethylene bis-(4tert-butylpyridinium chloride) (SAD-128) and decamethonium on reactivation of soman and sarininhibited cholinesterase by oximes, Biochem. Pharmacol. 27 (1978) 757-761.

[62] J. Bajgar, Prophylaxis against organophosphorus poisoning, J. Med. Che. Def. 1 (2004) 1-16.

[63] E. Reiner, Inhibition of acetylcholinesterase by 4,4'-bipyridine and its effect upon phosphylation of the enzyme, Croat. Chem. Acta 59 (1986) 925-931.

[64] G. Lallement, V. Baille, D. Baubichon, P. Carpentier, J.M. Collombet, P. Filliat, A. Foquin, E. Four, C. Masqueliez, G. Testylier, L. Tonduli, F. Dorandeu, Review of the value of huperzine as pretreatment of organophosphate poisoning, Neurotoxicology 23 (2002) 1-5.

[65] S. Eckert, P. Eyer, H. Muckter, F. Worek, Kinetic analysis of the protection afforded by reversible inhibitors against irreversible inhibition of acetylcholinesterase by highly toxic organophosphorus compounds, Biochem. Pharmacol. 72 (2006) 344-357.

[66] M.A. Dunn, B.E. Hackley, F.R. Sidell, Pretreatment for nerve agent exposure, in: F.R. Sidell, E.T. Takafuji, D.R. Franz (Eds.), Medical Aspects of Chemical Biological Warfare. Walter Reed Army Medical Center, Washington, 1997, pp. 181-196.

[67] D.E. Lenz, C.A. Broomfield, D.M. Maxwell, D.M. Cerasoli,. Nerve agent bioscavengers: protection against high- and low-dose organophosphorus exposure, in: S.M. Somani, J.A. Romano Jr. (Eds.), Chemical Warfare Agents: Toxicity at Low Levels. CRS Press LLC, Boca Raton, Florida, 2001, pp. 215-243.

[68] L. Raveh, E. Grauer, J. Grunwald, E. Cohen, Y. Ashani, The stoichiometry of protection against soman and VX toxicity in monkeys pretreated with human butyrylcholinesterase, Toxicol. Appl. Pharmacol. 145 (1997) 43-53.

[69] P. Masson, F. Nachon, C. A. Broomfield, D. E. Lenz, L. Verdier, L.M. Schopfer, O. Lockridge, A collaborative endeavor to design cholinesterase-based catalytic scavengers against toxic organophosphorus esters. Chem. Biol. Interact. 175 (2008) 273-280.

[70] A. Lucić Vrdoljak, M. Čalić, B. Radić, S. Berend, D. Jun, K. Kuča, Z. Kovarik, Pretreatment with pyridinium oximes improves antidotal therapy against tabun poisoning, Toxicology 228 (2006) 41-50. 


\section{Table 1}

Reversible inhibition of human recombinant acetylcholinesterase (AChE) and horse serum butyrylcholinesterase (BChE) by $N$-substituted 2-hydroxyiminoacetamides 1-4.

\begin{tabular}{|c|c|c|c|c|}
\hline $\begin{array}{l}N \text {-substituted } 2 \text { - } \\
\text { hydroxyiminoacetamide }\end{array}$ & $\begin{array}{l}{[\mathrm{ATCh}] /} \\
\mathrm{mmol} / \mathrm{L}\end{array}$ & $\begin{array}{c}{[N \text {-substituted } 2-} \\
\text { hydroxyiminoacetamide }] / \\
\mu \mathrm{mol} / \mathrm{L}\end{array}$ & $K_{\mathrm{i}} / \mu \mathrm{mol} / \mathrm{L}$ & $\alpha$ \\
\hline \multicolumn{5}{|l|}{$\mathrm{AChE}$} \\
\hline 1 & $0.1-0.6$ & $500-1500$ & $1187 \pm 248$ & $2.5 \pm 1.0$ \\
\hline 2 & $0.1-0.6$ & $30-150^{\mathrm{a}}$ & $358 \pm 197$ & $0.9 \pm 0.7$ \\
\hline 3 & $0.1-0.8$ & $15-80$ & $49 \pm 22$ & $2.9 \pm 1.5$ \\
\hline 4 & $0.1-0.8$ & $50-200$ & $135 \pm 26$ & $4.8 \pm 1.3$ \\
\hline \multicolumn{5}{|l|}{$\mathrm{BChE}$} \\
\hline 1 & $0.1-0.8$ & $50-200$ & $132 \pm 14$ & $6.3 \pm 2.2$ \\
\hline 2 & $0.1-0.8$ & $15-75$ & $42 \pm 4$ & $15 \pm 10$ \\
\hline 3 & $0.1-0.8$ & $0.1-0.4$ & $0.33 \pm 0.03$ & $6.0 \pm 1.7$ \\
\hline 4 & $0.1-0.8$ & $20-100$ & $30 \pm 3$ & $5.7 \pm 1.8$ \\
\hline
\end{tabular}

\footnotetext{
${ }^{\mathrm{a}}$ Due to the low solubility of 2 in $0.1 \mathrm{M}$ sodium phosphate buffer, $\mathrm{pH} 7.4$, the constant could not be evaluated at higher concentration of $\mathbf{2}$.
} 


\section{Table 2}

List of $N$-substituted 2-hydroxyiminoacetamide-enzyme interactions.

\begin{tabular}{|c|c|c|}
\hline Enzyme & $\begin{array}{l}N \text {-substituted 2- } \\
\text { hydroxyiminoacetamide }\end{array}$ & Interactions $^{\mathrm{a}}$ \\
\hline \multicolumn{3}{|l|}{$\overline{\mathrm{AChE}}$} \\
\hline & 1 & D) Tyr133 \\
\hline & & A) Ser203, His447 \\
\hline & & $\pi-\pi) \operatorname{Trp} 86$ \\
\hline & 2 & D) Tyr124, Ser203, Tyr337 \\
\hline & & $\begin{array}{l}\text { A) none } \\
\pi-\pi) \operatorname{Trp} 86\end{array}$ \\
\hline & 3 & D) none \\
\hline & & A) Glu202 \\
\hline & & $\pi-\pi)$ Tyr124, Trp286, Phe297 ( $\pi$-sigma), Tyr341 \\
\hline & 4 & D) Ser203, Phe295 \\
\hline & & A) Glu202 \\
\hline & & $\pi-\pi) \operatorname{Trp} 86, \operatorname{Tyr} 341(\pi$-sigma $)$ \\
\hline \multicolumn{3}{|r|}{ 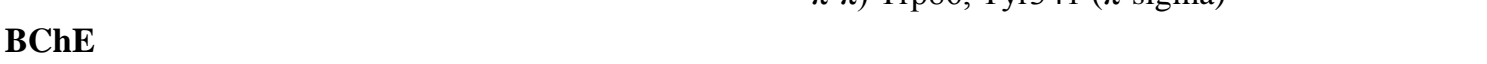 } \\
\hline & 1 & $\begin{array}{l}\text { D) Ser198, } \\
\text { A) Ser198, } \\
\pi-\pi) \operatorname{Trp} 82\end{array}$ \\
\hline & 2 & D) Ser198 \\
\hline & & A) Leu286 \\
\hline & & $\pi-\pi)$ Trp82, Phe329 \\
\hline & 3 & D) Gly116, Gly117 \\
\hline & & A) Ser198, Pro285 \\
\hline & & $\pi-\pi) \operatorname{Trp} 82$, His 438 \\
\hline & 4 & D) Thr120 \\
\hline & & A) $\operatorname{Trp} 82$ \\
\hline & & $\pi-\pi) \operatorname{Trp} 82, \operatorname{Tyr} 332$ \\
\hline
\end{tabular}

$\left.{ }^{\mathrm{a}} \mathrm{D}\right) \mathrm{H}$-bond donor; A) H-bond acceptor; $\pi-\pi$ interactions. 

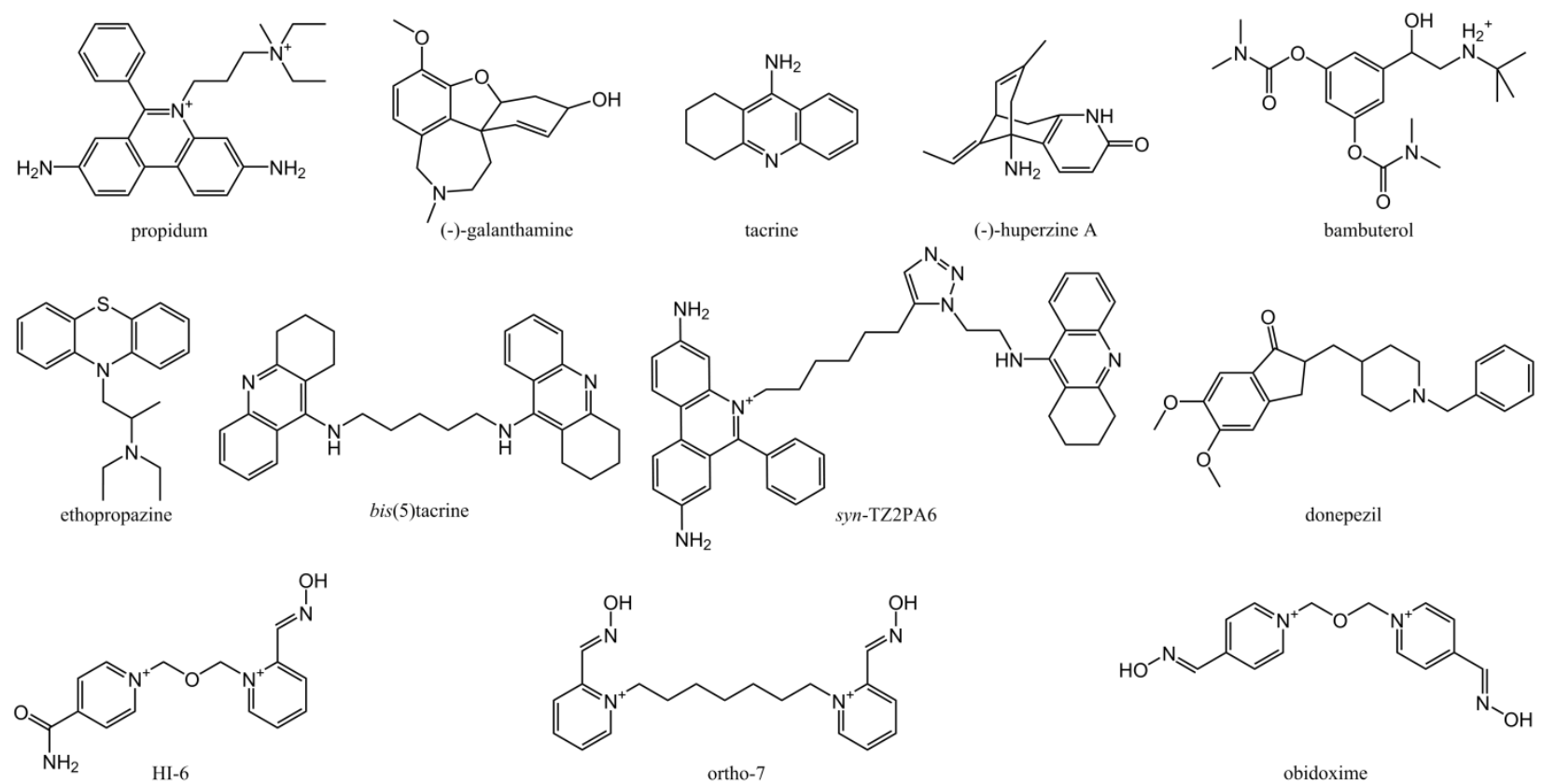

Fig. 1. Structures of cholinesterase inhibitors, and reactivators of organophosphorus nerve agentinhibited cholinesterases. 
<smiles>N#CCC(NC(=O)/C=N/O)c1ccccc1</smiles>
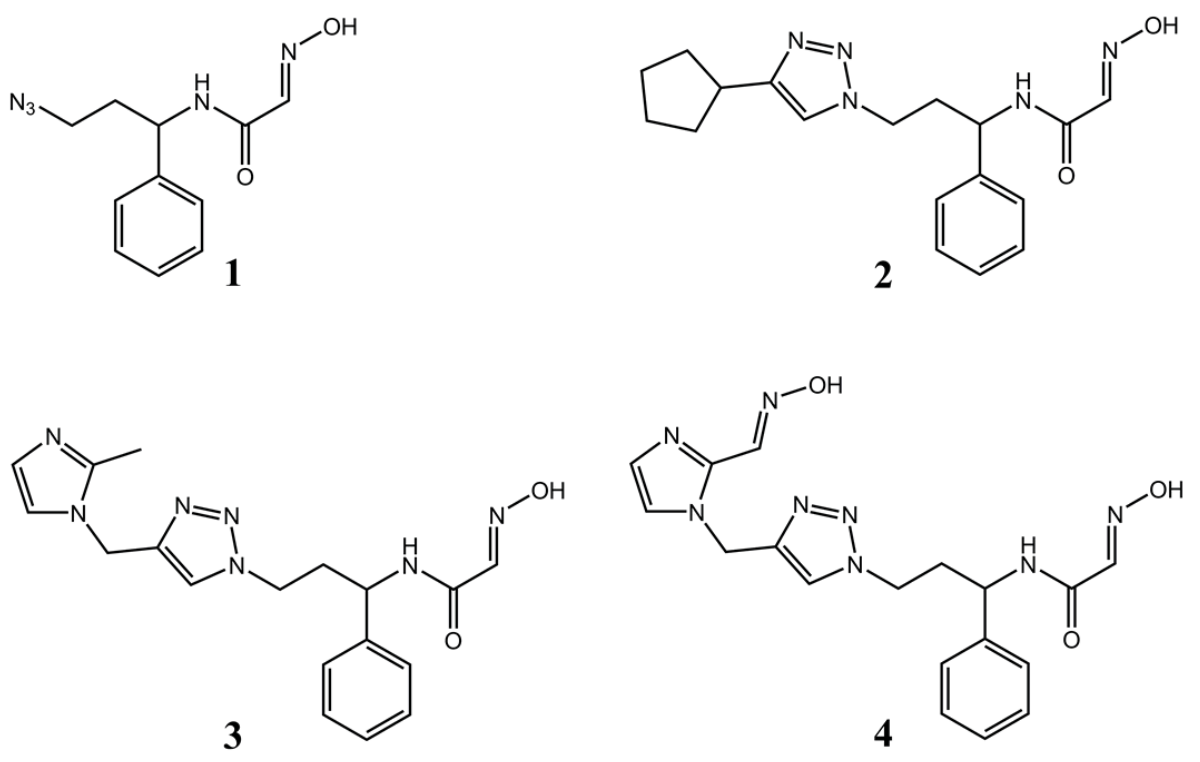

Fig. 2. Chemical structures of synthesized $N$-substituted 2-hydroxyiminoacetamides.
24

25

26

27

28

29

30

31

32

33

34

35

36

37

38

39

40

41

42

43

44

45

46

47

48

49

50

51

52

53

54

55

56

57

58

59

60

61

62

63

64

65 


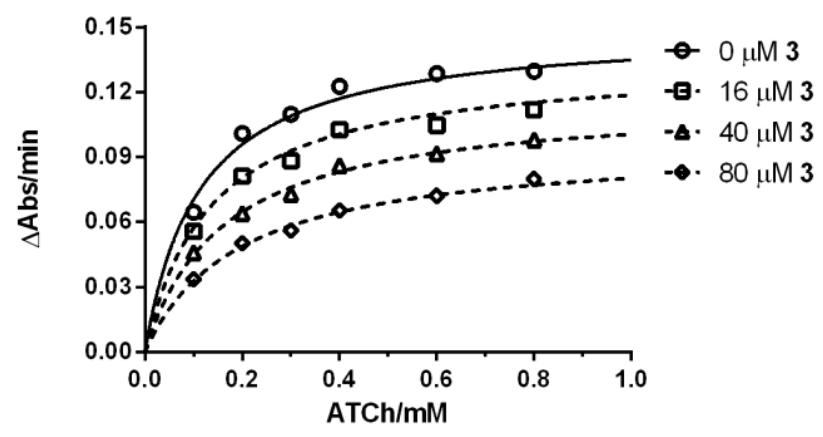

Fig. 3. Representative plot of AChE activity and the effect of substrate concentration on AChE activity in the presence and absence of $N$-substituted 2-hydroxyiminoacetamide 3 . To limit the influence of DMSO on the degree of enzyme inhibition the final content of DMSO was held constant at $0.1 \%$. 


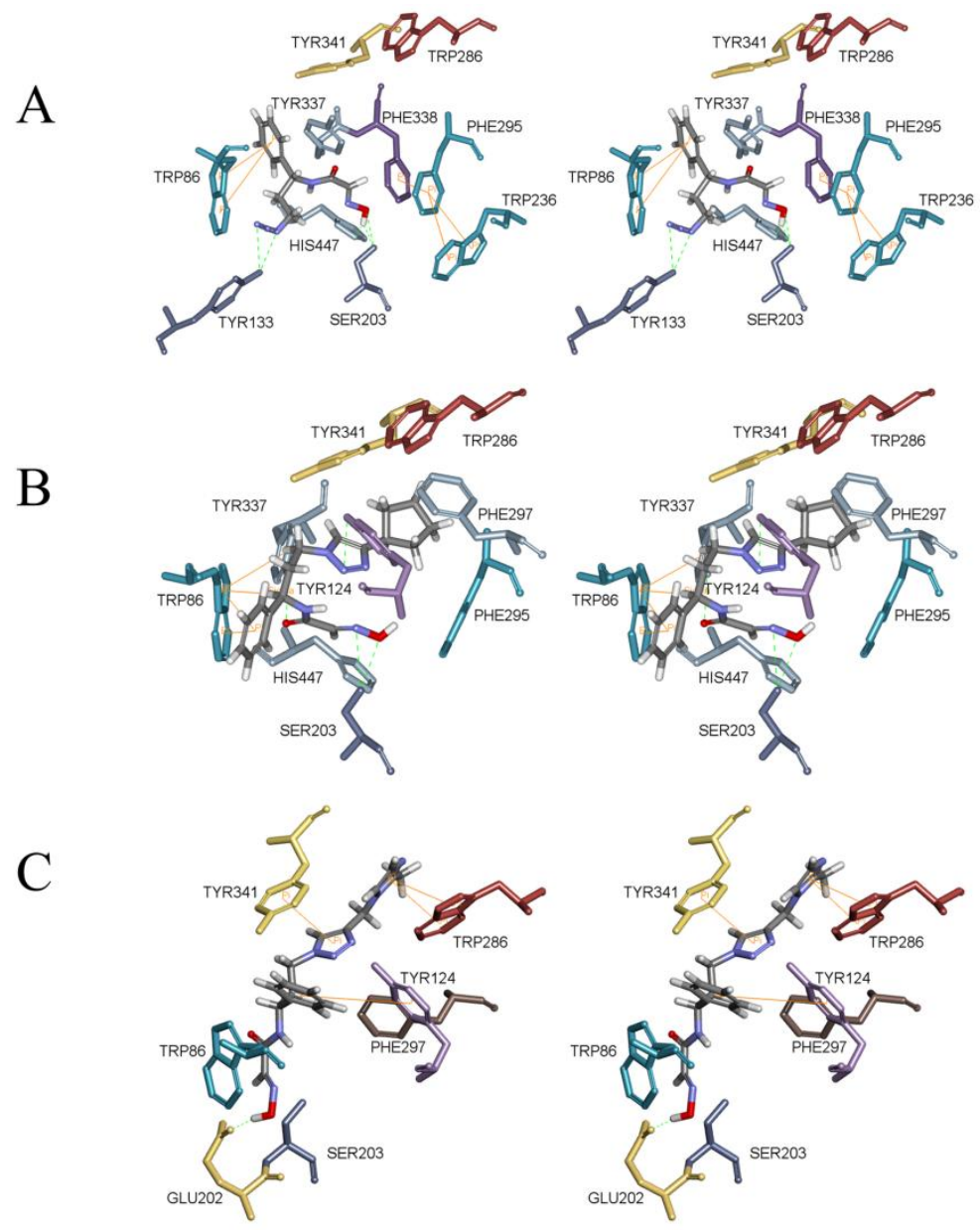

$\mathrm{D}$
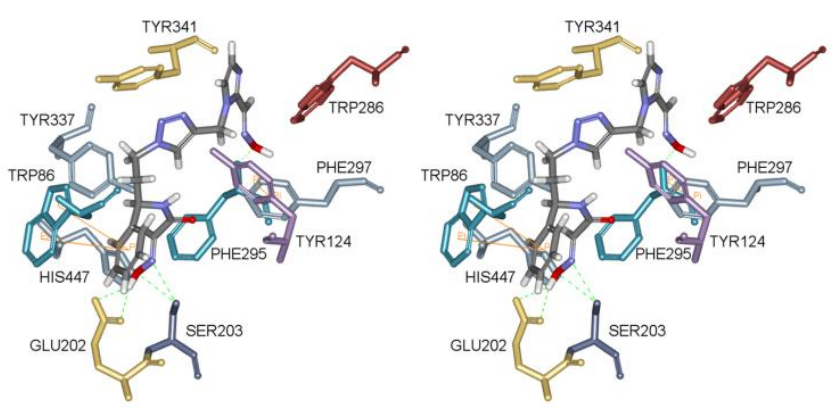

Fig. 4. The stereo view of docked conformation of the $N$-substituted 2-hydroxyiminoacetamides 1-4 (A-D, respectively) in the active site of the AChE. Non-covalent interactions are shown as green dashed lines (H-bonds) and orange lines $(\pi$ interactions). 
A
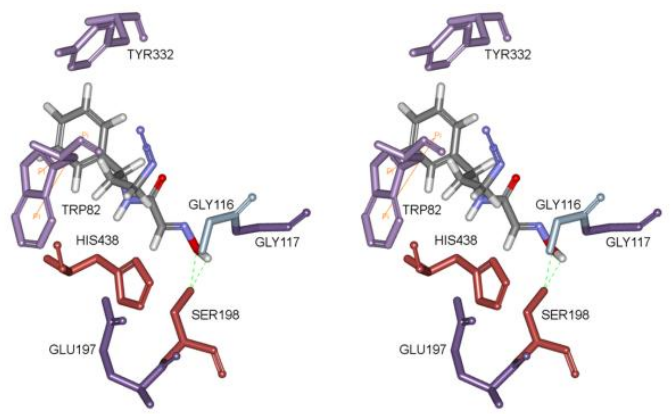

B
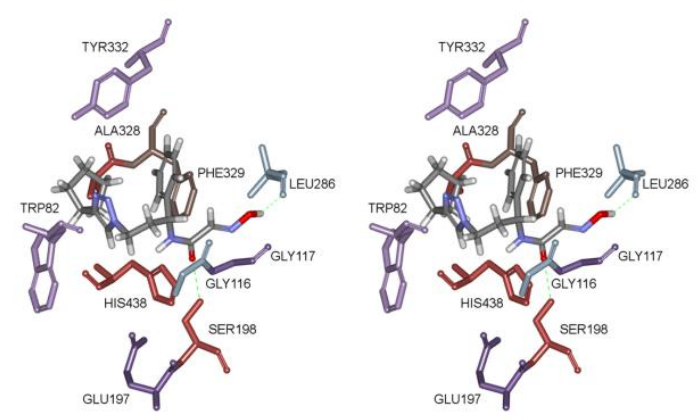

C
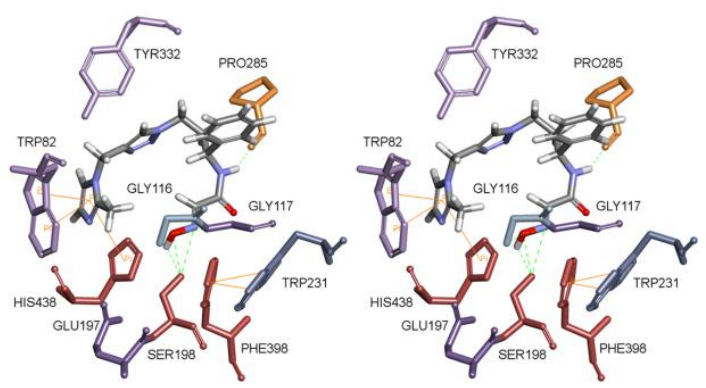

D
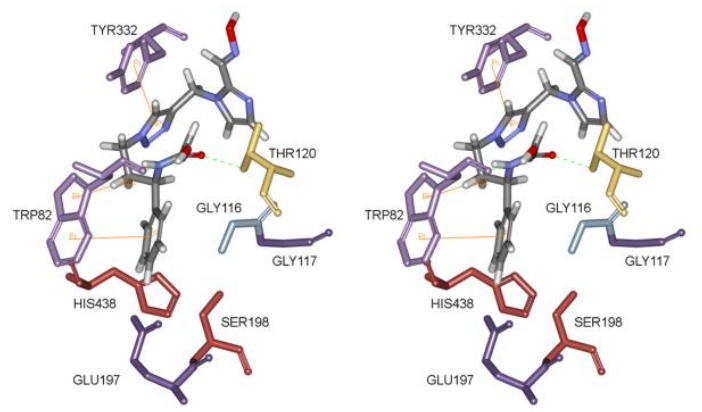

Fig. 5. The stereo view of docked conformation of the $N$-substituted 2-hydroxyiminoacetamides 1-4 (A-D) in the active site of the BChE. Non-covalent interactions are shown as green dashed lines (H-bonds) and orange lines ( $\pi$ interactions). 